\title{
Differences in TGF- $\beta 1$ signaling and clinicopathologic characteristics of histologic subtypes of gastric cancer
}

\author{
Kyung Ho Pak ${ }^{1,3}$, Dong Hoon Kim² ${ }^{2}$ Hyunki Kim ${ }^{4}$, Do Hyung Lee ${ }^{5}$ and Jae-Ho Cheong ${ }^{5,6,7,8 *}$
}

\begin{abstract}
Background: Aberrant TGF- $\beta 1$ signaling is suggested to be involved in gastric carcinogenesis. However, the role of TGF- $\beta 1$ in intestinal-type $[i-G C]$ and diffuse-type $[d-G C]$ gastric cancer remains largely unknown. In this study, we evaluated the expression of TGF- $\beta 1$ signaling molecules and compared the clinicopathological features of $\mathrm{i}-\mathrm{GC}$ and $\mathrm{d}-\mathrm{GC}$.

Methods: Patients ( $n=365$, consecutive) who underwent curative gastrectomy for gastric adenocarcinoma in 2005 were enrolled. We performed immunohistochemical staining of TGF- $\beta 1$, TGF- $\beta 1$ receptor-2 (TRR2), Smad4, p-ERK1/2, TGF-activated kinase (TAK) 1, and p-Akt in 68 paraffin-embedded tumor blocks (33 i-GC and $35 \mathrm{~d}$-GC), scored the expression according to the extent of staining, and evaluated differences between the histologic subtypes.

Results: Patients with d-GC differed from those with i-GC as follows: younger and more likely to be female; more aggressive stage; higher recurrence rate. The expression of TGF- $\beta 1$ and T $\beta R 2$ was higher in i-GC $(P=0.05$ and $P<0.001$, respectively). The expression of Smad4, a representative molecule of the Smad-dependent pathway, was decreased in both subtypes. TAK1 and p-Akt, two major molecules involved in the Smad-independent pathway, were over-expressed (69 $87 \%$ of cases stained), without a statistically significant difference between i-GC and d-GC. Of note, the expression of p-ERK1/2, a Smad-independent pathway, was significantly increased in i-GC $(P=0.008)$.
\end{abstract}

Conclusions: The clinicopathological characteristics vary in different histologic gastric cancer subtypes. Although TGF- $\beta 1$ signaling in gastric cancer cells appears hyper-activated in i-GC compared to d-GC, the Smad-dependent pathway seems down-regulated while the Smad-independent pathway seems up-regulated in both histologic subtypes.

Keywords: TGF- $\beta 1$, Lauren classification, Gastric cancer

\section{Background}

In 2012, gastric cancer was responsible for 723,000 deaths and was ranked as the world's third leading cause of cancer mortality [1]. Gastric cancer was also the second most common malignancy in Korea [2]. Although conventional chemotherapy has improved the overall prognosis of gastric cancer, the survival rate of patients with advanced cancer still falls short of expectations. With the recent advances in our understanding of the molecular basis of this deadly disease, deregulated

\footnotetext{
* Correspondence: jhcheong@yuhs.ac

${ }^{5}$ Depatment of Surgery, Yonsei University College of Medicine, 50-1

Yonsei-ro, Seodaemun-gu 120-752Seoul, Korea

${ }^{6}$ Department of Biochemistry \& Molecular Biology, Yonsei University College of Medicine, Seoul, Korea

Full list of author information is available at the end of the article
}

cellular pathways were identified and targeted, providing new therapeutic options beyond conventional chemotherapies. Indeed, human epidermal growth factor receptor 2 (HER2) and vascular endothelial growth factor receptor 2 (VEGFR2) have been evaluated as therapeutic targets and are now available as treatment targets in metastatic gastric cancer [3, 4].

Data from one multicenter transcriptome study [5] and The Cancer Genome Atlas [6] established the significance of transforming growth factor beta 1 (TGF)- $\beta 1$ signaling on gastric cancer progression, supporting its role as an emerging candidate biomarker for gastric cancer. In line with these pivotal studies, others also showed the relation between high expression of TGF- $\beta 1$ and unfavorable prognosis of gastric cancer patients [7-10]. 
TGF- $\beta 1$ has an important role not only in normal physiologic functions like embryonic development, angiogenesis, fibrosis, and wound healing, but also in cancer development and progression. Furthermore, TGF- $\beta 1$ has a dual role in cancer, as a tumor suppressor in earlier stages and tumor promoter in later stages [11]. TGF- $\beta 1$ signaling falls into either the canonical Smaddependent pathway or non-canonical Smad-independent pathway. The Smad-independent pathway includes the phosphoinositol-3 kinase (PI3K), mitogen-activated protein kinase (MAPK), and small guanosine triphosphatase (GTPase) pathways. These pathways are most often implicated in tumor cell motility and migration [12].

Gastric cancer is characterized by tumor heterogeneity. The Lauren classification is a well-known histologic classification system used in gastric cancer [13]. According to the Lauren classification, gastric cancer is categorized into intestinal (i-GC), diffuse (d-GC), or mixed types. Typically, i-GC tumors form gastric gland-like structures, while d-GCs do not. In addition to morphologic differences, these two types are known to have different epidemiologic, clinical, and molecular manifestations as well [14].

Our study aimed to investigate the expression pattern of TGF- $\beta 1$ pathway-related molecules, including Smaddependent and Smad-independent factors, in gastric cancers of different Lauren's classifications.

\section{Methods}

\section{Patients and samples}

A series of 774 gastric cancer patients, who have undergone curative gastrectomy at Yonsei University Medical University Hospital (Seoul, Korea) from May 2005 to December 2005, were enrolled in this study. Patients who had a history of concurrent tumor, neoadjuvant chemotherapy or radiotherapy, preoperatively or intraoperatively detected metastasis, unavailable Lauren's data, and follow-up loss were excluded from this study. The clinicopathologic data of the remaining 365 patients who satisfied the inclusion criteria were analyzed to investigate the clinicopathological differences between iGC and d-GC. Patients were followed up clinically for at least 5 years after surgery, except in mortality cases. The follow-up time ranged from 3 to 69 months, with an average follow-up time of 55 months. We performed an analysis of mRNA microarray in the cases of 158 tissues among 365 cases as a pilot study. We observed that increased expression of TGF- $\beta$ pathway gene modules were associated with unfavorable survival from that analysis (data not shown). Based on the results, further experiment of immunohistochemical study were planned to validate the clinical meaning of TGF- $\beta$ signaling in gastric cancer subtypes. For more precise analysis, we decided to match the cases with considering the underlying clinic-pathological characteristics between the two subtypes of gastric cancer which might affect the interpretation of our results. A total of 68 tissues among 158 cases, therefore, were selected after some cases were dropped out because of the problems of tissue availability and readability. Written informed consent was obtained from all patients and the study was approved by the Institutional Review Board of Severance Hospital, Yonsei University (4-2012-0427).

\section{Immunohistochemistry}

Paraffin-embedded sections (4 $\mu \mathrm{m}$ thick) were deparaffinized with xylene and rehydrated in decreasing concentrations of ethanol. Sections were then incubated with $3 \% \mathrm{H}_{2} \mathrm{O}_{2}$ for $30 \mathrm{~min}$ at room temperature. The slides were immersed in $0.01 \mathrm{M}$ citrate buffer $(\mathrm{pH}$ 6.0) for $10 \mathrm{~min}$ for antigen retrieval and then immersed in phosphate-buffered saline (PBS) containing $15 \%$ goat serum. The primary antibodies were rabbit anti-human TGF- $\beta 1$ polyclonal antibody (1:100; Santa Cruz, USA), mouse anti-human Smad4 monoclonal antibody (1:100; Santa Cruz, USA), mouse anti-human TGF- $\beta$ receptor II (TßRII) monoclonal antibody (1:100; Santa Cruz, USA), rabbit anti-human phosphorylated (p)-Akt monoclonal antibody (1:100; Abcam, UK), rabbit anti-human pextracellular signal-regulated kinase (ERK) $1 / 2$ polyclonal antibody (1:100; Santa Cruz, USA) and rabbit anti-human TGF-activated kinase 1 (TAK1) polyclonal antibody (1:100; Abcam, UK). After rinsing with PBS, secondary antibodies were added (goat anti-rabbit polymerized horse radish peroxidase [HRP]-labeled secondary antibody or goat anti-mouse IgG secondary antibody; Envision kit, DAKO, Denmark), and the slides placed in a thermostatic water bath at $37^{\circ} \mathrm{C}$ for $30 \mathrm{~min}$. After rinsing with PBS, the samples were counterstained using hematoxylin.

\section{Evaluation of results of immunohistochemical staining}

Tissue samples were scored independently by two researchers (H.K. and K.H.P), who were blinded to the clinical data. Staining results for TGF- $\beta 1, T \beta R 2$, Smad4, TAK1, p-ERK1/2, and p-Akt were classified by estimating the percentage of epithelial cells exhibiting specific immunoreactivity: negative (no immunoreactivity); weak ( 0 to $33 \%$ positive cells); moderate (33 to $67 \%$ positive cells); and strong ( $>67 \%$ positive cells) (Fig. 1). Only samples exhibiting moderate and strong immunoreactivity were considered positive. We used following cells as internal positive controls: inflammatory cells for TGF- $\beta 1$ and p-Erk1/2; endothelial cells for T $\beta R 2$ and Smad4; Myocytes for p-Akt1. For TAK1, it was difficult to find internal positive control (Additional file 1: Figure S1).

\section{Statistical analysis}

Statistical analyses were performed using the SPSS statistical software program for Windows version 21 (SPSS 


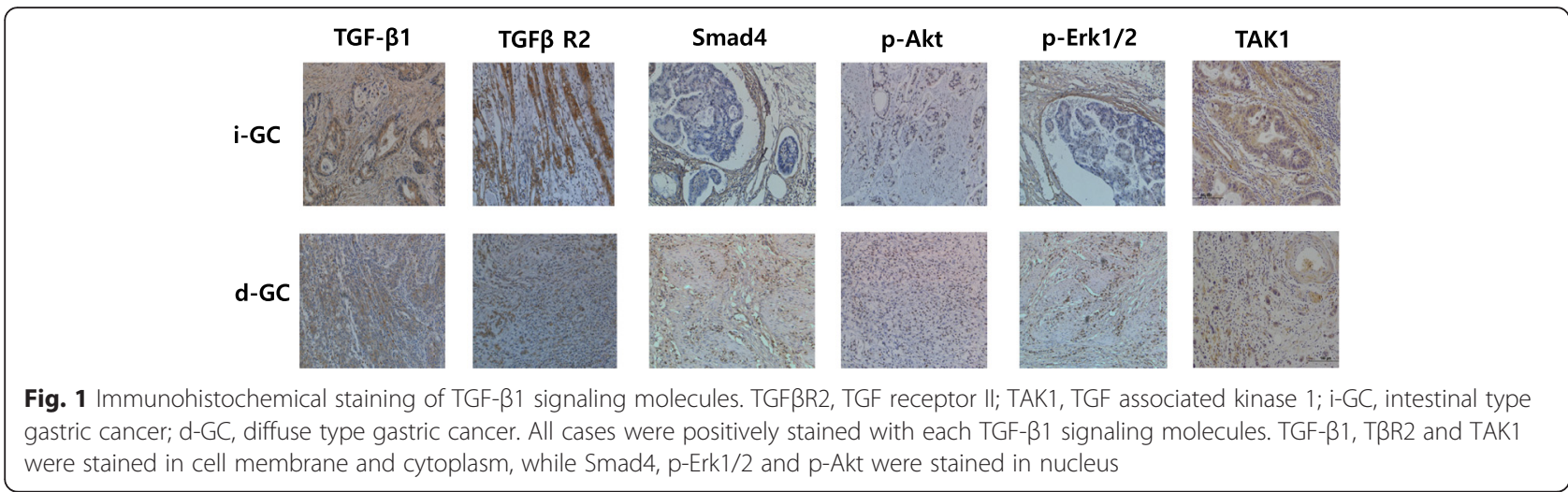

Inc., Chicago, IL, USA). The association between variables was tested using Pearson's $\chi^{2}$ or Fisher's exact tests for categorical variables. The survival data were analyzed using the Kaplan-Meier method and the log-rank test was used for assessing differences between groups. Twosided values of $P<0.05$ were considered to indicate statistically significant differences.

\section{Results}

\section{Differences in clinicopathologic features according to} Lauren's classification

The clinicopathologic data for patient groups sorted based on Lauren's classification are summarized in Tables 1 and 2. Patients with d-GC were significantly younger $(P<0.001)$ and more likely to be female $(P<0.001)$.
A larger proportion of the d-GC patients underwent total gastrectomy with more upper tumor location $(P=0.001)$ than i-GC patients. Patients with d-GC also had an increased propensity for recurrent disease $(P=0.028)$, in particular, peritoneal recurrence $(P=0.008)$. The overall survival rate of $\mathrm{d}-\mathrm{GC}$ patients showed an unfavorable trend compared to that of $\mathrm{i}-\mathrm{GC}$ patients $(P=0.072)$.

Tumors in patients with d-GC were larger $(P=$ $0.021)$, more undifferentiated $(P<0.001)$, and of higher T- $(P<0.001)$ and N-stages $(P=0.004)$ than those in i-GC patients. Lymphovascular invasion (LVI) was not different between the two patient groups $(P=0.099)$.

After selecting stage matched gastric cancer patients according to Lauren's classification (Table 3), none of

Table 1 Differences in demographic features of gastric cancer patients according to Lauren's classification

\begin{tabular}{|c|c|c|c|c|}
\hline & & Intestinal-type & Diffuse-type & $P$-value \\
\hline & & $(n=204)$ & $(n=161)$ & \\
\hline \multirow[t]{2}{*}{ Age } & $<60$ years & 86 & 113 & $<0.001^{*}$ \\
\hline & $>60$ years & 118 & 47 & \\
\hline \multirow[t]{2}{*}{ Sex } & Male & 163 & 99 & $<0.001^{*}$ \\
\hline & Female & 41 & 62 & \\
\hline \multirow[t]{2}{*}{ Extent of resection } & TG & 30 & 47 & $0.001^{*}$ \\
\hline & STG & 174 & 114 & \\
\hline \multirow[t]{3}{*}{ Location } & Fundus & 16 & 48 & $0.001^{*}$ \\
\hline & Body & 67 & 79 & \\
\hline & Antrum & 117 & 63 & \\
\hline \multirow[t]{2}{*}{ Recurrence } & $(-)$ & 172 & 122 & $0.028^{*}$ \\
\hline & $(+)$ & 32 & 39 & \\
\hline \multirow[t]{4}{*}{ Recurrence site } & Hematogenous & 11 & 4 & $0.008^{*}$ \\
\hline & Peritoneal & 11 & 27 & \\
\hline & Locoregional & 3 & 4 & \\
\hline & Mixed & 7 & 3 & \\
\hline \multirow[t]{2}{*}{ Survival } & Alive & 175 & 126 & 0.072 \\
\hline & Dead & 29 & 35 & \\
\hline
\end{tabular}

${ }^{*} P<0.05$, calculated using; N.S. not significant, TG total gastrectomy, STG subtotal gastrectomy 
Table 2 Differences in pathological characteristics according to Lauren's classification

\begin{tabular}{|c|c|c|c|c|}
\hline & & $\begin{array}{l}\text { Intestinal-type } \\
(n=204)\end{array}$ & $\begin{array}{l}\text { Diffuse-type } \\
(n=161)\end{array}$ & $P$-value \\
\hline \multirow[t]{2}{*}{ Size } & $<30 \mathrm{~mm}$ & 134 & 88 & $0.021^{*}$ \\
\hline & $>30 \mathrm{~mm}$ & 70 & 73 & \\
\hline \multirow[t]{2}{*}{ Differentiation } & Differentiated & 188 & 4 & $<0.001^{*}$ \\
\hline & Poorly differentiated & 16 & 157 & \\
\hline \multirow[t]{2}{*}{ LVI } & $(-)$ & 140 & 97 & 0.099 \\
\hline & $(+)$ & 64 & 64 & \\
\hline \multirow[t]{4}{*}{ T stage } & T1 & 126 & 70 & $<0.001^{*}$ \\
\hline & $\mathrm{T} 2$ & 17 & 13 & \\
\hline & T3 & 33 & 19 & \\
\hline & T4 & 28 & 59 & \\
\hline \multirow[t]{4}{*}{ N stage } & No & 146 & 92 & $0.004^{*}$ \\
\hline & N1 & 22 & 14 & \\
\hline & N2 & 11 & 17 & \\
\hline & N3 & 25 & 38 & \\
\hline
\end{tabular}

${ }^{*} P<0.05$, calculated using; N.S. not significant, $L V /$ lymphovascular invasion

the parameters between i-GC and d-GC patients showed statistically significant differences, with the exception of patient age (younger in $\mathrm{d}-\mathrm{GC} ; P<0.01$ ).

\section{Expression of TGF- $\beta 1$ signaling molecules}

Positive expression rate of TGF- $\beta 1$ signaling molecules in $\mathrm{i}-\mathrm{GC}$ and $\mathrm{d}-\mathrm{GC}$, respectively, was as follows; TGF- $\beta 1$ (61 and 34\%), TßR2 (100 and $66 \%$ ), Smad4 (26 and $27 \%$ ), p-ERK1/2 (82 and $37 \%)$, p-Akt (72 and $69 \%$ ), and TAK1 (87 and 83 \%) (Fig. 1; Expression data is summarized in Table 4).

Table 3 Clinicopathologic characteristics of 68 randomly selected gastric cancer patients whose tumors were utilized for immunohistochemical analysis

\begin{tabular}{|c|c|c|c|c|}
\hline & & $\begin{array}{l}\text { Intestinal-type } \\
(n=33)\end{array}$ & $\begin{array}{l}\text { Diffuse-type } \\
(n=35)\end{array}$ & $P$-value \\
\hline \multirow[t]{2}{*}{ Age } & $<60$ years & 10 & 25 & $<0.01$ \\
\hline & $>60$ years & 23 & 10 & \\
\hline \multirow[t]{2}{*}{ Sex } & Male & 24 & 25 & NS \\
\hline & Female & 9 & 10 & \\
\hline \multirow[t]{2}{*}{ Depth of invasion } & $\mathrm{T} 1$ & 9 & 10 & NS \\
\hline & $\geq \mathrm{T} 2$ & 24 & 25 & \\
\hline \multirow[t]{2}{*}{ LN metastasis } & Negative & 17 & 20 & NS \\
\hline & Positive & 16 & 15 & \\
\hline \multirow[t]{3}{*}{ TNM stage* } & 1 & 8 & 11 & NS \\
\hline & $\|$ & 14 & 16 & \\
\hline & III & 11 & 8 & \\
\hline
\end{tabular}

* $7^{\text {th }}$ ed., N.S. not significant
TGF- $\beta 1$ and T $\beta R 2$ were activated in both gastric cancer histologic subtypes. However, all the TGF- $\beta 1$ signaling pathway molecules were expressed at higher levels in i-GC than in d-GC tumors $(P=0.05, P<0.001$ in all $)$. The co-expression rate of TGF- $\beta$ ligand and receptor was much higher in $\mathrm{i}-\mathrm{GC}(20 / 33,64.5 \%)$ than in $\mathrm{d}-\mathrm{GC}$ tumors $(11 / 37,35.5 \% ; P=0.015)$. The expression of Smad4, a representative molecule of the Smad-dependent pathway, was decreased in both subtypes, albeit without statistically significant difference between the subtypes

Table 4 Expression of TGF- $\beta$ signaling molecules according to Lauren's classification

\begin{tabular}{|c|c|c|c|c|}
\hline & & $\begin{array}{l}\text { Intestinal-type } \\
(n=33)\end{array}$ & $\begin{array}{l}\text { Diffuse-type } \\
(n=35)\end{array}$ & $\begin{array}{l}P- \\
\text { value }\end{array}$ \\
\hline \multirow[t]{2}{*}{ TGF- $\beta 1$} & Low & 13 & 23 & 0.050 \\
\hline & High & 20 (61 \%) & 12 (34 \%) & \\
\hline \multirow[t]{2}{*}{$\mathrm{T} \beta \mathrm{R} 2$} & Low & 0 & 12 & $<0.001$ \\
\hline & High & 33 (100\%) & $23(66 \%)$ & \\
\hline \multirow[t]{2}{*}{ Smad4 } & Low & 23 & 25 & 0.588 \\
\hline & High & $8(26 \%)$ & $9(27 \%)$ & \\
\hline \multirow[t]{2}{*}{$p-E R K 1 / 2$} & Low & 3 & 12 & 0.008 \\
\hline & High & $14(82 \%)$ & 7 (37 \%) & \\
\hline \multirow[t]{2}{*}{ p-Akt } & Low & 9 & 11 & 0.796 \\
\hline & High & $23(72 \%)$ & $24(69 \%)$ & \\
\hline \multirow[t]{2}{*}{ TAK1 } & Low & 4 & 6 & 0.758 \\
\hline & High & 27 (87 \%) & $29(84 \%)$ & \\
\hline
\end{tabular}

TGF- $\beta 1$ transforming growth factor- $\beta 1, T \beta R 2$ TGF- $\beta$ receptor $2, p$-ERK phosphorylated extracellular signal-regulated kinase, TAK1 TGF-activated kinase1 
$(P=0.588)$. Among factors involved in the Smadindependent pathway, p-Akt and TAK1 were overexpressed, without a statistically significant difference between i-GC and d-GC $(P=0.796$ and $P=0.742$, respectively). Of note, the expression of p-ERK1/2 was higher in $\mathrm{i}-\mathrm{GC}$ than in $\mathrm{d}-\mathrm{GC}(P=0.008)$.

Further, the increased TGF- $\beta 1$ signaling pathway was significantly correlated with poor overall survival in gastric cancer patients $(50.2 \pm 5.2$ months vs. $63.8 \pm$ 1.8 months, $P=0.03$ ) (Fig. 2).

\section{Discussion}

In this study, we identified significant clinicopathologic differences between i-GC and d-GC. Our study could support the possibility of the conversion of TGF- $\beta 1$ signaling from a Smad-dependent to a Smad-independent pathway in case of malignant status, a hypothesis suggested in one previous study [15]. In addition, we propose that gastric cancer subtypes may utilize a different Smad-independent signaling pathway. Our data suggest that i-GC might depend more on the p-ERK1/2 pathway than d-GC. It can be supported by the previous study [16] which reported that p-ERK1/2 pathway is associated with $H$. pylori infection- well known proven cause of i-GC [17].

TGF- $\beta 1$ has the potential to function as a tumor suppressor (via its effects on proliferation, replication potential, and apoptosis), or as a tumor promoter (via its effects on migration, invasion, angiogenesis, and the immune system) [11]. Based on animal models and in vitro studies, Elliot and Blobe [18] proposed a hypothesis, in which during early tumorigenesis, TGF- $\beta 1$-mediated tumor suppressor activity functions through a Smaddependent pathway, while tumor promoter activity acts through a Smad-independent pathway. However, no complete clinical study included empirical data supporting this hypothesis, except that of Kim et al. [15]. Their analysis of a 332-tissue microarray, which was performed along the normal epithelium-atrophic gastritis-dysplasia-carcinoma sequence, showed that TGF- $\beta 1$ and T $\beta R 1$ expression continually increased along this sequence, while Smad $2 / 3$ and Smad4 decreased as carcinoma progressed. Their study, however, was limited because they did not evaluate the expression of Smad-independent signaling molecules. In our present study, we evaluated two TGF- $\beta 1$ pathways in gastric cancers by assessing the expression of Smad -dependent and Smad -independent signaling molecules, although we did not compare the expression of TGF- $\beta 1$ signaling molecules between tumor and normal tissue. When considering our data and the study of Kim et al. [15], we suggest a model for the role of TGF- $\beta$ in gastric carcinogenesis, whereby TGF- $\beta 1$ signaling changes from the tumor-suppressive Smad-dependent pathway to a tumor-activating Smad-

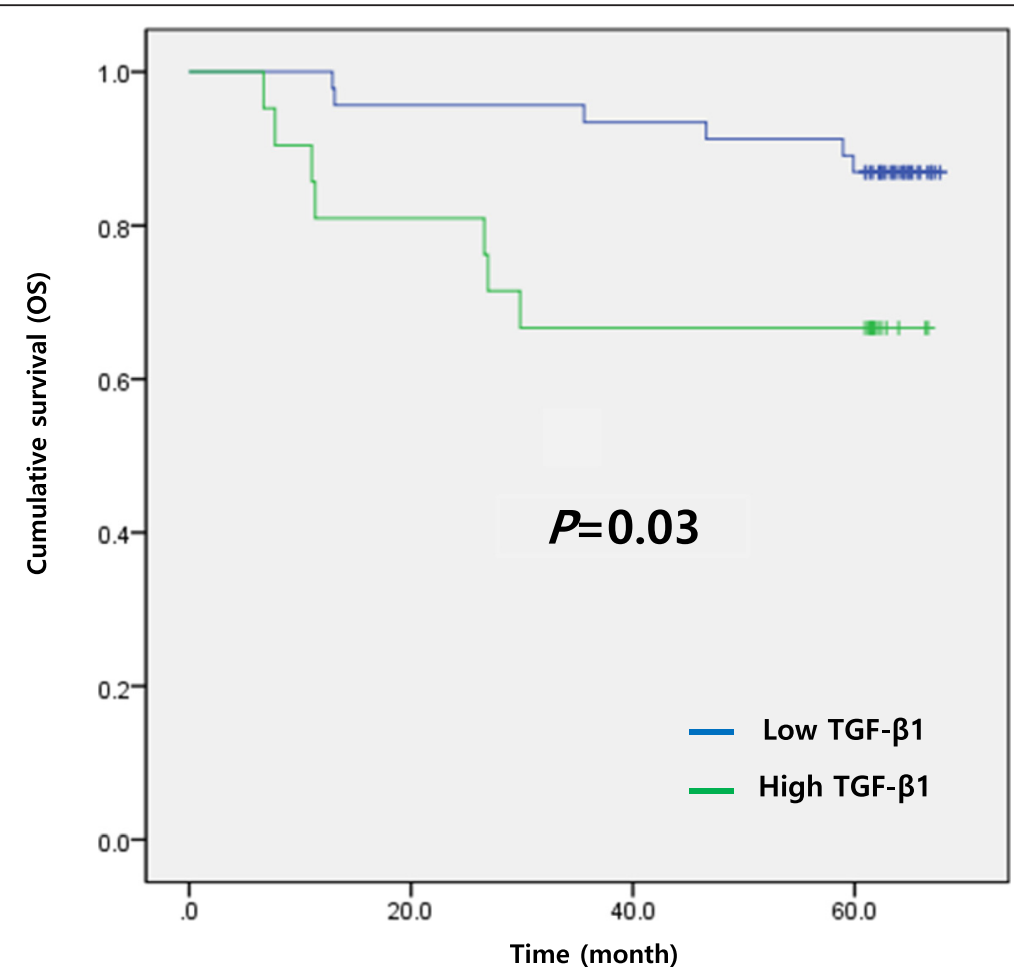

Fig. 2 Kaplan-Meier analysis of overall survival between high TGF- $\beta 1$ signal and low TGF- $\beta 1$ signal. Patients group with high TGF- $\beta 1$ showed poorer overall survival (50.2 \pm 5.2 months vs. $63.8 \pm 1.8$ months, $p=0.03)$ 
independent pathway as the cancer progresses, irrespective of histologic subtypes of gastric cancer.

The main sources of TGF- $\beta 1$ are stromal cells, such as fibroblasts, lymphocytes, and macrophages [12]. Therefore, the expression of TGF- $\beta 1$ within tumors is higher in $\mathrm{d}-\mathrm{GC}$ than in $\mathrm{i}-\mathrm{GC}$, largely because $\mathrm{d}-\mathrm{GC}$ has more stromal components [5]. However, our study, in which we assessed the immunoreactivity of only cancer cells not stroma, revealed another interesting aspect of TGF$\beta$ signaling in gastric cancer; the expression of ligand and receptor of TGF- $\beta 1$ signaling was increased in gastric cancer tissue, which suggests a potential autocrine loop in gastric cancer. Interestingly, in our data, coexpression of both ligand and receptor was higher in iGC than in $\mathrm{d}-\mathrm{GC}$. It is thus conceivable that a paracrine effect of TGF- $\beta 1$ signaling is dominant in $d-G C$, in which cancerous stromal cells are abundant, while an autocrine function of TGF- $\beta 1$ might play an important role in $\mathrm{i}-\mathrm{GC}$. We will address this hypothesis in our future studies.

We note the limitations of our current study; our sample of gastric cancer tissues was relatively small and had a possibility of selection bias. In addition, we did not compare the expression of TGF- $\beta 1$ signaling molecules in cancer tissues with that in matched normal tissues.

Therapeutic strategies targeting TGF- $\beta 1$ signaling in cancer treatment is a burgeoning field of research [19]. The current results can augment our understanding of the role of TGF- $\beta 1$ signaling in distinct histologic subtypes of gastric cancer.

\section{Conclusions}

The clinicopathological characteristics vary in different histologic gastric cancer subtypes. Although TGF- $\beta 1$ signaling in gastric cancer cells appears hyper-activated in i-GC compared to d-GC, the Smad-dependent pathway seems down-regulated while the Smad-independent pathway seems up-regulated in both histologic subtypes. Of note, the expression of $\mathrm{p}$-ERK1/2, a Smadindependent pathway factor, was significantly increased in $\mathrm{i}-\mathrm{GC}$.

\section{Additional file}

Additional file 1: Figure S1. Following cells were used as internal positive controls: inflammatory cells for TGF- $\beta 1$ and p-Erk1/2; endothelial cells for T $\beta R 2$ and Smad4; Myocytes for p-Akt1. For TAK1, it was difficult to find internal positive control. (PPTX $13592 \mathrm{~kb}$ )

\section{Abbreviations}

i-GC: intestinal type gastric cancer; d-GC: diffuse type gastric cancer; LVI: Iymphovascular invasion; TAK1: TGF associated kinase 1.

\section{Competing interests}

There is no disclosure of any commercial interest that the authors may have in the subject of study or the source of any financial or material support.

\section{Authors' contributions}

JC was guarantor of integrity of the entire study, designed the research. KHP analyzed the data and drafted the manuscript. KHP, DHL and DHK participated in the experiments of immunohistochemical staining. HK and DHK counted the immunoreactivity of samples. All authors have read and approved in final manuscript.

\section{Acknowledgements}

This work was supported by a grant of the Korea Health Technology R\&D Project (grant No. HI13C2162) through the Korea Health Industry

Development Institute (KHIDI), the National R\&D Program for Cancer Control (No. 1020390) funded by the Ministry of Health \& Welfare, Republic of Korea, and by Grant No. HURF-2014-45 from the Hallym University Research Fund.

\section{Author details}

${ }^{1}$ Department of Surgery, Hallym University Medical Center, Hwasung, Korea. ${ }^{2}$ Department of Pathology, Hallym University Medical Center, Hwasung, Korea. ${ }^{3}$ Department of Medicine, Yonsei University Graduate School, Seoul, Korea. ${ }^{4}$ Department of Pathology, Yonsei University College of Medicine, Seoul, Korea. ${ }^{5}$ Depatment of Surgery, Yonsei University College of Medicine, 50-1 Yonsei-ro, Seodaemun-gu 120-752Seoul, Korea. ${ }^{6}$ Department of Biochemistry \& Molecular Biology, Yonsei University College of Medicine, Seoul, Korea. ${ }^{7}$ Brain Korea 21 PLUS Project for Medical Science, Yonsei University College of Medicine, Seoul, Korea. ${ }^{8}$ Open NBI Convergence Technology Research Laboratory, Yonsei University College of Medicine, Seoul, Korea.

Received: 5 August 2015 Accepted: 28 January 2016

Published online: 04 February 2016

References

1. Ferlay J, Soerjomataram I, Dikshit R, Eser S, Mathers C, Rebelo M, et al. Cancer incidence and mortality worldwide: Sources, methods and major patterns in GLOBOCAN 2012. Int J Cancer. 2014;136:E359-86.

2. Jung KW, Won YJ, Kong HJ, Oh CM, Cho H, Lee DH, et al. Cancer statistics in Korea: incidence, mortality, survival, and prevalence in 2012. Cancer Res Treat. 2015:47(2):127-41.

3. Bang YJ, Van Cutsem E, Feyereislova A, Chung HC, Shen L, Sawaki A, et al. Trastuzumab in combination with chemotherapy versus chemotherapy alone for treatment of HER2-positive advanced gastric or gastrooesophageal junction cancer (ToGA): a phase 3, open-label, randomised controlled trial. Lancet. 2010;376(9742):687-97.

4. Fuchs CS, Tomasek J, Yong CJ, Dumitru F, Passalacqua R, Goswami C, et al. Ramucirumab monotherapy for previously treated advanced gastric or gastro-oesophageal junction adenocarcinoma (REGARD): an international, randomised, multicentre, placebo-controlled, phase 3 trial. Lancet. 2014:383(9911):31-9.

5. Wu Y, Grabsch H, Ivanova T, Tan IB, Murray J, Ooi CH, et al. Comprehensive genomic meta-analysis identifies intra-tumoural stroma as a predictor of survival in patients with gastric cancer. Gut. 2013;62(8):1100-11.

6. Cancer Genome Atlas Research Network. Comprehensive molecular characterization of gastric adenocarcinoma. Nature. 2014;513(7517):202-9.

7. Kinugasa S, Abe S, Tachibana M, Hishikawa Y, Yoshimura H, Monden N, et al. Overexpression of transforming growth factor-beta1 in scirrhous carcinoma of the stomach correlates with decreased survival. Oncology. 1998;55(6):582-7.

8. Maehara Y, Kakeji Y, Kabashima A, Emi Y, Watanabe A, Akazawa K, et al. Role of transforming growth factor-beta 1 in invasion and metastasis in gastric carcinoma. J Clin Oncol. 1999;17(2):607-14.

9. Kim YH, Lee HS, Lee HJ, Hur K, Kim WH, Bang YJ, et al. Prognostic significance of the expression of Smad4 and Smad7 in human gastric carcinomas. Ann Oncol. 2004;15(4):574-80.

10. Hawinkels LJ, Verspaget HW, van Duijn W, van der Zon JM, Zuidwijk K Kubben FJ, et al. Tissue level, activation and cellular localisation of TGFbeta1 and association with survival in gastric cancer patients. $\mathrm{Br} J$ Cancer. 2007;97(3):398-404. 
11. Achyut BR, Yang L. Transforming growth factor $\beta$ in the gastrointestinal and hepatic tumor microenvironment. Gastroenterology. 2011;141(4):1167-78.

12. Ikushima H, Miyazono K. TGFbeta signalling: a complex web in cancer progression. Nat Rev Cancer. 2010;10(6):415-24.

13. Lauren P. The two histological main types of gastric carcinoma: diffuse and so-called intestinal-type carcinoma. An attempt at a histo-clinical classification. Acta Pathol Microbiol Scand. 1965;64:31-49.

14. Yuasa Y. Control of gut differentiation and intestinal-type gastric carcinogenesis. Nat Rev Cancer. 2003;3(8):592-600.

15. Kim S, Lee S, Choi Y, Wang L, Park CK, Shin YK. Extensive alteration in the expression profiles of TGFB pathway signaling components and TP53 is observed along the gastric dysplasia-carcinoma sequence. Histol Histopathol. 2008;23(12):1439-52.

16. Zhu Y, Zhong X, Zheng S, Du Q, Xu W. Transformed immortalized gastric epithelial cells by virulence factor CagA of Helicobacter pylori through Erk mitogen-activated protein kinase pathway. Oncogene. 2005;24(24):3886-95.

17. Uemura N, Okamoto S, Yamamoto S, Matsumura N, Yamaguchi S, Yamakido $M$, et al. Helicobacter pylori infection and the development of gastric cancer. N Engl J Med. 2001;345(11):784-9.

18. Elliott RL, Blobe GC. Role of transforming growth factor Beta in human cancer. J Clin Oncol. 2005;23(9):2078-93.

19. Akhurst RJ, Hata A. Targeting the TGFbeta signalling pathway in disease. Nat Rev Drug Discov. 2012;11(10):790-811.

\section{Submit your next manuscript to BioMed Central} and we will help you at every step:

- We accept pre-submission inquiries

- Our selector tool helps you to find the most relevant journal

- We provide round the clock customer support

- Convenient online submission

- Thorough peer review

- Inclusion in PubMed and all major indexing services

- Maximum visibility for your research

Submit your manuscript at www.biomedcentral.com/submit 